\title{
Optimal Infinite-Horizon Feedback Laws for a General Class of Constrained Discrete-Time Systems: Stability and Moving-Horizon Approximations
}

\author{
S. S. KEERTHI ${ }^{3}$ AND E. G. GILBERT ${ }^{2}$
}

Communicated by J. L. Speyer

\begin{abstract}
Stability results are given for a class of feedback systems arising from the regulation of time-varying discrete-time systems using optimal infinite-horizon and moving-horizon feedback laws. The class is characterized by joint constraints on the state and the control, a general nonlinear cost function and nonlinear equations of motion possessing two special properties. It is shown that weak conditions on the cost function and the constraints are sufficient to guarantee uniform asymptotic stability of both the optimal infinite-horizon and movinghorizon feedback systems. The infinite-horizon cost associated with the moving-horizon feedback law approaches the optimal infinite-horizon cost as the moving horizon is extended.
\end{abstract}

Key Words. Discrete-time systems, infinite-horizon optimal control, moving-horizon control, state-control constraints, nonquadratic cost functions, stability.

\section{Introduction}

Consider the following discrete-time system with constraints:

$$
\begin{aligned}
& x_{k+1}=f_{k}\left(x_{k}, u_{k}\right), \quad y_{k}=g_{k}\left(x_{k}, u_{k}\right), \quad k \geq i, \\
& \left(x_{k}, u_{k}\right) \in Z_{k} \subset R^{n} \times R^{m}, \quad k \geq i, \quad x_{i}=a,
\end{aligned}
$$

where, for $k \geq 0, f_{k}: R^{n} \times R^{m} \rightarrow R^{n}$ and $g_{k}: R^{n} \times R^{m} \rightarrow R^{\prime}$. For $k \geq 0$, let $h_{k}: R^{l} \times R^{m} \rightarrow R$ be a nonnegative function. Our problem is to determine a feedback law $u_{k}=\eta_{k}\left(x_{k}\right), k \geq 0$, which, for each $i \geq 0$ and feasible initial

\footnotetext{
${ }^{1}$ Assistant Professor, School of Automation, Indian Institute of Science, Bangalore, India.

${ }^{2}$ Professor, Department of Aerospace Engineering, University of Michigan, Ann Arbor, Michigan.
} 
state $a \in R^{n}$, generates through (1) a sequence $\left\{u_{k}\right\}_{k \geq i}$ that minimizes the cost to go

$$
J_{i}=\sum_{k=i}^{\infty} h_{k}\left(y_{k}, u_{k}\right)
$$

subject to (1) and (2). The constraint set $Z_{k}$ and the functions $f_{k}, g_{k}$, and $h_{k}$ satisfy

$$
\begin{aligned}
& (0,0) \in Z_{k}, \quad f_{k}(0,0)=0, \quad g_{k}(0,0)=0, \\
& h_{k}(0,0)=0, \quad k \geq 0
\end{aligned}
$$

Thus, we have an output regulator problem where the targets for $x_{k}, y_{k}$, and $u_{k}$ are the origins. The state regulator problem is a special case of the output regulator problem in which $g_{k}(x, u) \equiv x$. The assumption (4) is not terribly restrictive, because many interesting problems can be made to satisfy it with a simple change of variables.

The prior literature is mostly concerned with stochastic regulator problems. See, for instance, Ref. 1. Deterministic problems are quite different in nature and previous results appear to be limited to the linear quadratic regulator problem (LQRP), where the following conditions hold:

(a) $f_{k}(x, u)=A_{k} x+B_{k} u, \quad g_{k}(x, u)=C_{k} x+D_{k} u, \quad k \geq 0$,

and the matrices $A_{k}, B_{k}, C_{k}$, and $D_{k}$ are uniformly bounded on $k \geq 0$;

(b) the sequence pairs $\left\{A_{k}, B_{k}\right\}_{k \geq 0}$ and $\left\{C_{k}, A_{k}\right\}_{k \geq 0}$ are, respectively, uniformly completely controllable and uniformly completely observable;

(c) $h_{k}(y, u)=y^{\prime} Q_{k} y+u^{\prime} R_{k} u, k \geqslant 0$, where prime indicates transpose and $Q_{k}$ and $R_{k}$ are symmetric positive-definite matrices satisfying, for some $\lambda_{2} \geq \lambda_{1}>0$,

$$
\begin{array}{ll} 
& \lambda_{1} y^{\prime} y \leq y^{\prime} Q_{k} y \leq \lambda_{2} y^{\prime} y, \quad \lambda_{1} u^{\prime} u \leq u^{\prime} R_{k} u \leq \lambda_{2} u^{\prime} u, \\
& k \geq 0, \quad(y, u) \in R^{\prime} \times R^{m}
\end{array}
$$

For the LQRP, it is known (for $D_{k} \equiv 0$ ) that the optimal feedback law is linear and that the resulting feedback system is exponentially stable (Ref. 2).

In this paper, we extend the results for the LQRP to the wider class of problems described by (1)-(3). To obtain our results, the system (1) must satisfy two special controllability and observability properties ( $\mathrm{C}$ and $\mathrm{O}$ ), which for the linear system (5) are implied, respectively, by uniform complete controllability and uniform complete observability. Under weak conditions on $h_{k}$ and $Z_{k}$, we show that an optimal feedback law exists and that the optimal feedback system is uniformly asymptotically stable; also, we 
give conditions which guarantee exponential stability of the optimal system. The known stability results for the LQRP are obtained by a simple application of our results. Our proofs are based on a standard Lyapunov stability theorem. Specialized to the LQR.P, they do not utilize the discrete-time Riccati equation or the linearity of the feedback law; hence, the proofs are more basic than the usual proofs used for the LQRP.

Even if (5) holds, there are at least two practical motivations for considering (1)-(3): the need to impose rigorous constraints on the state and/or control, the possibility that nonquadratic cost functions may lead to a more desirable quality of regulation. Unless (1)-(3) is a time-invariant LQRP, it is usually impossible to characterize analytically or compute the optimal feedback law $\eta_{k}$. This leads naturally to the moving-horizon feedback law, $\hat{\eta}_{k}$, which is a computationally feasible approximation of $\eta_{k}$.

The moving-horizon feedback law plays a key role in our work. In the literature, it is also referred to as the receding-horizon feedback law. The moving-horizon feedback law was first considered by Kleinman (Ref. 3) as an easy tool for stabilizing a linear time-invariant system. However, it was Thomas and Barraud (Refs. 4 and 5) who added a new insight into Kleinman's method by recognizing the underlying notion of the moving terminal set. Their results applied to linear time-invariant systems and the cost function

$$
h_{k}(y, u)=u^{\prime} R u, \quad k \geq 0, \quad(y, u) \in R^{\prime} \times R^{m} .
$$

Kwon and Pearson (Refs. 6 and 7) extended the ideas to the LQRP and were the first to employ the moving-horizon feedback law as a means of approximating the infinite-horizon feedback law, $\eta_{k}$. More recently, Kwon et al. (Ref. 8) have considered certain computational issues related to the feedback laws considered in Ref. 6 . Little has been done on moving-horizon feedback laws for problems with state-control constraints, general cost functions, and nonlinear system dynamics. A preliminary treatment of some of the results in this paper appears in Refs. 9 and 10.

The moving-horizon feedback law for (1)-(3) is defined as follows. For $k \geq 0$, let $M_{k}$ be a positive integer denoting the moving-horizon at index $k$. When the system is in operation at time index $i \geq 0$, let its state be $x_{i}$. To determine the moving-horizon control $u_{i}$, define $a=x_{i}$. Solve the optimal control problem of minimizing

$$
\hat{J}_{i}=\sum_{k=i}^{i+M_{i}-1} h_{k}\left(y_{k}, u_{k}\right)
$$

subject to (1), (2) and the alternative constraints

$$
i \leq k \leq i+M_{i}-1, \quad x_{i+M_{i}}=0 .
$$


Let

$$
\left\{\hat{u}_{k}\left(i, a ; M_{i}\right)\right\}_{k=i}^{i+M_{i}-1}
$$

be an optimal control sequence for this problem. Then, choose $u_{i}=$ $\hat{u}_{i}\left(i, a ; M_{i}\right)$. In other words, the moving-horizon feedback law at index $i$ is defined by the function $\hat{\eta}_{i}\left(\because M_{i}\right)$, where $\hat{\eta}_{i}\left(a ; M_{i}\right)=\hat{u}_{i}\left(i, a ; M_{i}\right)$.

Except for special problems, it is not possible to give a formula for $\hat{\eta}_{k}$. However, in principle $\hat{\eta}_{k}$ can be computed as follows. Given $i \geq 0$ and $a=x_{i}$, state the finite-dimensional optimization problem defined by $(1),(2)$, (6), and (7) as a large-scale mathematical programming problem whose independent variables are the components of $x_{i}, \ldots, x_{i+M_{i}-1}$ and $u_{i}, \ldots, u_{i+M_{i}-1}$. Solve the mathematical programming problem by using one of the well established techniques. Then, obtain $\hat{\eta}_{i}\left(a ; M_{i}\right)$ as indicated in the previous paragraph. We will refer to such a procedure as feedback by on-line mathematical programming (FOMP). The advance of computer technology makes it possible to entertain the notion of the extensive on-line computations. Recently, on-line procedures similar to the FOMP idea have been investigated by Knudsen (Ref. 11) and DeVlieger et al. (Ref, 12) for minimum-time problems and by Gutman (Ref. 13) for regulator problems with $l_{1}$-cost functions. However, only special examples are treated and there is no general theory.

If the $h_{k}$ and $Z_{k}$ satisfy the same requirements as for the infinite-horizon problem, and if the $M_{k}, k \geq 0$, satisfy certain weak conditions, we show that a moving-horizon feedback law exists and that the feedback system resulting from the moving-horizon feedback law enjoys the same stability properties as the optimal infinite-horizon feedback system. Moreover, by choosing the $M_{k}$ sufficiently large, the associated infinite-horizon cost $J_{i}$ in (3), associated with $u_{k}=\hat{\eta}_{k}\left(x_{k} ; M_{k}\right)$, can be made arbitrarily close to the optimal cost of the infinite-horizon control problem. The only prior results of a similar nature, obtained by Kwon and Pearson (Ref. 7) for the time-varying LQRP, follow easily from our results.

The organization of the paper is as follows. Section 2 introduces notations and slight generalizations of some standard stability definitions. Section 3 defines and discusses the two special properties which are required of system (1). Stability results for the optimal infinite-horizon and movinghorizon feedback laws are then stated in Sections 4 and 5 respectively. The relationship between the costs given by these feedback laws is described in Section 6. Because of the similarities in the optimal infinite-horizon and moving-horizon problems, it is more efficient and illuminating to bring the proofs together in one place; this we do in Section 7. Finally, Section 8 gives examples which illustrate some advantages that may accrue from applying the general problem formulation. 


\section{Notations and Definitions}

We begin with some notations and definitions. Given a column vector $x \in R^{p}$ and a matrix $M \in R^{q \times p}$, let

$$
\|x\|=\sqrt{x^{\prime} x} \text { and }\|M\|=\sup \{\|M x\|:\|x\|=1\} .
$$

We interpret $(x, y) \in R^{p} \times R^{q}$ as the single column $\left[x^{\prime}, y^{\prime}\right]^{\prime} . R_{+}$is the set of nonnegative reals. For $\in \in R_{+}$,

$$
N(\epsilon)=\{x:\|x\| \leq \epsilon\} .
$$

$I_{n}$ denotes the $n \times n$ identity matrix. A function $W: R_{+} \rightarrow R_{+}$is said to belong to class $\mathscr{K}_{\infty}$ if:

(a) it is continuous;

(b) $W(s)=0 \Leftrightarrow s=0$;

(c) it is nondecreasing;

(d) $W(s) \rightarrow \infty$ when $s \rightarrow \infty$.

Let $W_{1}, W_{2}$ belong to class $\mathscr{K}_{\infty}$ and define

$$
W_{3}(s)=W_{2}\left(W_{1}(s)\right), \quad W_{4}(s)=\max \left\{W_{1}(s), W_{2}(s)\right\} .
$$

It follows easily that $W_{3}, W_{4}$ are in class $\mathscr{K}_{\infty}$ and that

$$
W_{1}\left(s_{1}\right)+W_{2}\left(s_{2}\right) \leq 2 W_{4}\left(s_{1}+s_{2}\right), \quad s_{1} \in R_{+}, \quad s_{2} \in R_{+} .
$$

The following abbreviations will be used: $\mathrm{MH}=$ moving-horizon; $\mathrm{IH}=$ infinite-horizon.

Since we treat systems with a constrained state space, it is necessary to modify slightly the usual stability definitions (Refs. 14 and 15) which apply to unconstrained systems. Consider the system

$$
x_{k+1}=F_{k}\left(x_{k}\right), \quad x_{k} \in X_{k} \subset R^{n}, \quad k \geq 0,
$$

where $F_{k}: X_{k} \rightarrow X_{k+1}$. Define

$$
Y=\left\{(i, a): i \geq 0, a \in X_{i}\right\} .
$$

For $(i, a) \in Y$, let $x_{k}^{*}(i, a), k \geq i$, denote the solution of (9), given $x_{i}=a$. A state $x_{e} \in R^{n}$ is said to be an equilibrium state for (9) if $x_{e} \in X_{k}$ and $F_{k}\left(x_{e}\right)=x_{e}$ for all $k \geq 0$. Assume hereafter that $x=0$ is an equilibrium state for (9).

With these minor changes in set-up, the usual definitions for local stability apply. The equilibrium state $x=0$ is uniformly stable (US) if, given $\epsilon>0, \exists \delta(\epsilon)>0$ such that

$$
\left\|x_{k}^{*}(i, a)\right\| \leq \epsilon, \quad(i, a) \in Y, \quad k \geq i, \quad a \in N(\delta) .
$$


It is uniformly asymptotically stable (UAS) if:

(a) it is US;

(b) $\exists r>0$ and, for any $\sigma>0$, a positive integer $T(\sigma)$ such that

$$
\left\|x_{k}^{*}(i, a)\right\| \leq \sigma, \quad(i, a) \in Y, \quad k \geq i+T, \quad a \in N(r) .
$$

The following definitions concern global stability. The equilibrium state $x=0$ is uniformly asymptotically stable in the large (UASL) if:

(a) it is US;

(b) given $r>0, \exists B(r)>0$ such that

$$
\left\|x_{k}^{*}(i, a)\right\| \leq B, \quad(i, a) \in Y, \quad k \geq i, \quad a \in N(r) ;
$$

(c) for any $\sigma>0$ and $r>0, \exists$ a positive integer $T(\sigma, r)$ such that (10) holds.

The origin is exponentially stable (ES) if $\exists$ constants $\phi>0$ and $0 \leq \zeta<1$ such that

$$
\left\|x_{k}^{*}(i, a)\right\| \leq \phi(\zeta)^{k-i}\|a\|, \quad(i, a) \in Y, \quad k \geq i .
$$

If (11) holds, $\zeta$ is the degree of ES.

\section{Properties $\mathrm{C}$ and $\mathrm{O}$}

Since the properties of controllability and observability play a crucial role in the theory of the LQRP, it is not surprising that similar properties come up in the treatment of the more general problem (1)-(3). Properties $\mathrm{C}$ and $\mathrm{O}$ appear in Sections 4-7. They are important because they appear to summarize just those characteristics of the nonlinear system (1) which are essential for the stability of the IH and MH optimal feedback systems. Before stating the definitions of Properties $\mathrm{C}$ and $\mathrm{O}$, we consider several simple consequences of uniform complete controllability and observability in the linear system (1), (5). This adds some insight to the definitions and, in the case of linear systems, provides algebraic tests for the properties.

Given $i \geq j \geq 0$, let $\Phi(i, j)$ be the state-transition matrix defined by

$$
\begin{array}{ll}
\Phi(i, j)=I_{n}, & \text { for } i=j, \\
\Phi(i, j)=A_{i-1} A_{i-2} \cdots A_{j}, & \text { for } i>j .
\end{array}
$$

The following definition is standard (Ref. 2). 
Definition 3.1. The sequence pair $\left\{A_{k}, B_{k}\right\}_{k \geq 0}$ is uniformly completely controllable if $\exists \mu_{c}>0$ and a positive integer $N_{c}$ such that

$$
\begin{aligned}
G_{c}\left(i, N_{c}\right) & =\sum_{j=i}^{i+N_{c}-1} \Phi\left(i+N_{c}, j+1\right) B_{j} B_{j}^{\prime} \Phi^{\prime}\left(i+N_{c}, j+1\right) \\
& \geq \mu_{c} I_{n}, \quad i \geq 0 .
\end{aligned}
$$

The above definition is an algebraic condition on $\left\{A_{k}, B_{k}\right\}_{k \geq 0}$. An alternative and slightly weaker notion of uniform complete controllability for (1), (5) is a consequence of (12).

Theorem 3.1. Assume that, for $k \geq 0$, the matrices $A_{k}$ and $B_{k}$ are uniformly bounded and that $\left\{\boldsymbol{A}_{k}, \boldsymbol{B}_{k}\right\}_{k \geq 0}$ is uniformly completely controllable. Then, $\exists p_{c}>0$ such that: for all $i \geq 0, a \in R^{n}, \exists$ a sequence pair $\left\{\left(x_{k}, u_{k}\right)\right\}_{k \geq i}$ which satisfies (1), (5), $x_{i}=a,\left(x_{k}, u_{k}\right)=(0,0), k \geq i+N_{c}$, and

$$
\sum_{k=i}^{i+N_{c}-1}\left\|\left(x_{k}, u_{k}\right)\right\| \leq p_{c}\|a\| \text {. }
$$

This theorem is easily proved by using the least-squares control sequence which transfers $x_{i}=a$ to $x_{i+N_{c}}=0$. The details are omitted. Inequality (13) requires the existence of a motion to the origin in which the state and control sequences are bounded, uniformly in $i$, by a multiple of $\|a\|$. Property $C$ is simply a weak form of (13).

Definition 3.2. System (1) has property C if $\exists$ a positive integer $N_{c}$ and a $\mathscr{K}_{\infty}$ function $W_{c}$ such that: for all $i \geq 0, a \in R^{n}, \exists$ a sequence pair $\left\{\left(x_{k}, u_{k}\right)\right\}_{k \geq i}$ which satisfies $(1), x_{i}=a_{9}\left(x_{k}, u_{k}\right)=(0,0), k \geq i+N_{c}$, and

$$
\sum_{k=i}^{i+N_{\mathrm{c}}-1}\left\|\left(x_{k}, u_{k}\right)\right\| \leq W_{c}(\|a\|) \text {. }
$$

Remark 3.1. The appearance of $x_{k}$ in (14) can be eliminated if the $f_{k}$ satisfy some additional conditions. Suppose, e.g., $\exists$ a $\mathscr{K}_{\infty}$ function $F$ such that

$$
\left\|f_{k}(x, u)\right\| \leq F(\|(x, u)\|), \quad k \geq 0, \quad(x, u) \in R^{n} \times R^{m},
$$

and that in Definition 3.2, (14) is replaced by

$$
\sum_{k=i}^{i+N_{k}-1}\left\|u_{k}\right\| \leq \bar{W}_{c}(\|a\|)
$$

Then, (1) satisfies Property C. The argument goes as follows. Clearly,

$$
\left\|x_{k+1}\right\| \leq F\left(\left\|x_{k}\right\|+\left\|u_{k}\right\|\right), \quad k \geq i \text {. }
$$


Applying property (8) successively shows

$$
\left\|x_{k}\right\| \leq F_{k-i}\left(\|a\|+\sum_{j=i}^{k-1}\left\|u_{j}\right\|\right) \leq F_{k-i}\left(\|a\|+\bar{W}_{c}(\|a\|)\right), \quad k>i,
$$

where $F_{j}$ is in class $\mathscr{K}_{\infty}$ for $j \geq 1$. Using this inequality in the sum on the left side of (14) and applying (8) repeatedly proves the existence of a $\mathscr{K}_{\infty}$ function $W_{c}$ bounding the sum.

We now turn to Property O. First, consider the linear system (1), (5).

Definition 3.3. The sequence pair $\left\{C_{k}, A_{k}\right\}_{k \geq 0}$ is uniformly completely observable if $\exists \mu_{0}>0$ and a positive integer $N_{0}$ such that

$$
G_{0}\left(i, N_{0}\right)=\sum_{j=i}^{i+N_{0}-1} \Phi^{\prime}(j, i) C_{j}^{\prime} C_{j} \Phi(j, i) \geq \mu_{0} I_{n}, \quad i \geq 0 .
$$

Theorem 3.2. Assume that, for $k \geq 0$, the matrices $A_{k}, B_{k}, C_{k}$, and $D_{k}$ are uniformly bounded and that $\left\{C_{k}, A_{k}\right\}_{k \geq 0}$ is uniformly completely observable. Then, $\exists p_{0}>0$ such that: for all $i \geq 0$ and sequences $\left\{\left(x_{k}, y_{k}, u_{k}\right)\right\}_{k \geqq i}$ that satisfy (1) and (5),

$$
\sum_{k=i}^{i+N_{0}-1}\left\|\left(y_{k}, u_{k}\right)\right\| \geq p_{0}\left\|x_{i}\right\| \text {. }
$$

The proof of Theorem 3.2 is given in Section 9. Condition (17) is the model for Property O.

Definition 3.4. System (1) has Property O if $\exists$ a positive integer $N_{0}$ and a $\mathscr{K}_{\infty}$ function $W_{0}$ such that: for all $i \geq 0$ and sequences $\left\{\left(x_{k}, y_{k}, u_{k}\right)\right\}_{k \geq i}$ that satisfy (1),

$$
\sum_{k=i}^{i+N_{0}-1}\left\|\left(y_{k}, u_{k}\right)\right\| \geq W_{0}\left(\left\|x_{i}\right\|\right)
$$

For $u_{k}=0, k \geq i$, it is obvious that Property $\mathrm{O}$ is a uniform observability property; by observing the outputs $y_{k}$ for $i \leq k \leq i+N_{0}-1$, it is possible to determine a bound on the size of the initial state $x_{i}$. For the general nonlinear system (1), it is not surprising that our subsequent results require $u_{k}$ to appear in (18). Unlike the case of linear systems, it is impossible to characterize all the interactions between $\left\{x_{k}\right\}_{k \geq i}$ and $\left\{y_{k}\right\}_{k \geq i}$ by setting $u_{k}=0, k \geq i$.

Remark 3.2. Consider the state regulator problem in which $g_{k}(x, u) \equiv$ $x$. Then, Property $\mathrm{O}$ is satisfied trivially. 
It is clear from Theorems 3.1 and 3.2 that for the linear case (5), Properties $\mathrm{C}$ and $\mathrm{O}$ are implied, respectively, by uniform complete controllability and uniform complete observability. Therefore, Properties $\mathrm{C}$ and $\mathrm{O}$ can be tested easily, particularly when $A_{k}, B_{k}$, and $C_{k}$ are index invariant. For the nonlinear system (1), it is not obvious how to proceed and this is an interesting topic for further research.

\section{Infinite-Horizon Feedback System}

In this section, we formulate precisely the $1 \mathrm{H}$ optimal control problem and state theorems concerning the existence and stability of the corresponding optimal feedback system.

For $i \geq 0, a \in R^{n}$, let $\mathscr{P}(i, a)$ denote the problem of minimizing the cost $J_{i}$ in (3) subject to (1) and (2). A sequence $\left\{\left(x_{k}, y_{k}, u_{k}\right)\right\}_{k \geq i}$ is admissible to $\mathscr{P}(i, a)$ if it satisfies (1) and (2).

To state our results, a variety of assumptions will be needed. Let $Z=\bigcap_{k \geq 0} Z_{k}$.

Assumption A1. $0 \in$ interior $Z$.

Assumption A2. For each $k \geq 0, Z_{k}$ is closed; $f_{k}: R^{n} \times R^{m} \rightarrow R^{n}$, $g_{k}: R^{n} \times R^{m} \rightarrow R^{\prime}$ are continuous; and $h_{k}: R^{\prime} \times R^{m} \rightarrow R$ is lower semicontinuous.

Assumption A3. $\exists$ a $\mathscr{H}_{\infty}$ function $G$ such that

$$
\left\|g_{k}(x, u)\right\| \leq G(\|(x, u)\|), \quad k \geq 0, \quad(x, u) \in R^{n} \times R^{m} .
$$

Assumption A4. $\exists$ a $\mathscr{K}_{\infty}$ function $H_{1}$ such that

$$
h_{k}(y, u) \leq H_{1}(\|(y, u)\|), \quad k \geq 0, \quad(y, u) \in R^{\prime} \times R^{m} .
$$

Assumption A5. $\exists$ a $\mathscr{K}_{\infty}$ function $H_{2}$ such that

$$
h_{k}(y, u) \geq H_{2}(\|(y, u)\|), \quad k \geq 0, \quad(y, u) \in R^{\prime} \times R^{m} .
$$

Assumption A6. The following conditions hold: below (5);

(a) $f_{k}$ and $g_{k}$ are given by (5) and satisfy the assumptions immediately

(b) $\exists$ positive numbers $p_{1}, p_{2}$, and $q$ such that

$$
\begin{aligned}
& p_{1}\|(y, u)\|^{q} \leq h_{k}(y, u) \leq p_{2}\|(y, u)\|^{q}, \\
& k \geq 0, \quad(y, u) \in R^{l} \times R^{m} ;
\end{aligned}
$$


(c) for $k \geq 0, h_{k}: R^{l} \times R^{m} \rightarrow R$ is lower semicontinuous;

(d) $Z_{k}=R^{n} \times R^{m}, k \geq 0$.

In proving certain results, the following assumption will serve as an alternative for A5.

Assumption $\mathbf{A 5}^{\prime}$. The following conditions hold:

(a) $l=n, g_{k}(x, u)=x, k \geq 0,(x, u) \in R^{n} \times R^{m}$;

(b) $h_{k}(y, u) \geq H_{3}(\|y\|), k \geq 0,(y, u) \in R^{l} \times R^{m}$, where $H_{3} \in \mathscr{H}_{\infty}$;

(c) for $k \geq 0,\left\{u:(x, u) \in Z_{k}\right\} \subset \Omega_{k}$, where $\Omega_{k}$ is compact.

Remark 4.1. Assumption A6 implies Assumptions A1-A5 and refers to unconstrained problems with linear equations of motion and special bounds on the cost function $h_{k}$.

Remark 4.2. Assumptions A3 and A4 mainly concern the existence of bounds on $g_{k}(x, u)$ and $h_{k}(y, u)$ that are uniform with respect to $k$; otherwise, they are merely technical in nature. Suppose, e.g., that $g_{k}(x, u)=$ $g(x, u), \quad h_{k}(y, u)=h(y, u), k \geq 0,(x, y, u) \in R^{n} \times R^{l} \times R^{m}$, where $g: R^{n} \times$ $R^{m} \rightarrow R^{i}$ and $h: R^{I} \times R^{m} \rightarrow R$ are continuous. Then, Assumptions A3 and A4 are automatically satisfied. To see this, let

$$
G(s)=\max (s, \max \{\|g(x, u)\|:\|(x, u)\| \leq s\})
$$

and

$$
H_{1}(s)=\max (s, \max \{h(y, u):\|(y, u)\| \leq s\}) .
$$

It is not difficult to verify that $G$ and $H_{1}$ are in class $\mathscr{K}_{\infty}$ and satisfy (19) and (20). We also note that, if $f_{k}(x, u)=f(x, u), k \geq 0,(x, u) \in R^{n} \times R^{m}$, and $f: R^{n} \times R^{m} \rightarrow R^{n}$ is continuous, then the bounding function $F$ required in Remark 3.1 is given by

$$
F(s)=\max (s, \max \{\|f(x, u)\|:\|(x, u)\| \leq s\}) .
$$

Remark 4.3. Assumption A5 is implied by anothier condition which may be easier to verify. Specifically, assume that $\exists$ a function $\bar{h}: R^{l} \times R^{m} \rightarrow R$ such that

$$
h_{k}(y, u) \geq \bar{h}(y, u), \quad k \geq 0, \quad(y, u) \in R^{l} \times R^{m},
$$

and the following conditions hold:

(a) $\bar{h}$ is continuous and nonnegative;

(b) $\bar{h}(y, u)=0 \Leftrightarrow(y, u)=(0,0)$;

(c) $\bar{h}(y, u) \rightarrow \infty$, whenever $(y, u) \rightarrow \infty$. 
To see that Assumption A5 is implied by (23), define

$$
H_{2}(s)=\min \{\bar{h}(y, u):\|(y, u)\| \geq s\}, \quad s \geq 0 .
$$

Remark 4.4. Assumption A5 requires that $h_{k}$ include a suitable cost on the control (e.g. the term $u^{\prime} R_{k} u$ in the LQRP). Assumption A5' does not require a control cost and refers to the state regulator problem with a compactness constraint on the control.

For $i \geq 0$, let

$$
\begin{aligned}
X_{i}=\{a: \mathscr{P}(i, a) \text { has an admissible sequence } \\
\text { for which } \left.J_{i} \text { is finite }\right\}
\end{aligned}
$$

Set

$$
X=\bigcap_{i \geq 0} X_{i}
$$

The following theorem concerns properties of these sets and the existence of an optimal control. The existence is a direct consequence of the results in Ref. 16, which are reviewed in Section 10.

Theorem 4.1. Consider $\mathscr{P}(i, a)$ for each $i \geq 0$.

(i) $X_{i}$ is nonempty.

(ii) Assumption A1 and Property $\mathrm{C}$ imply $0 \in$ interior $X$.

(iii) $Z_{k}=R^{n} \times R^{m}, k \geq 0$, and Property C imply $X=R^{n}$.

(iv) Assumptions A2, A5 (or $\mathrm{A} 5^{\prime}$ ) and $a \in X_{i}$ imply $\mathscr{P}(i, a)$ has a solution.

(v) Suppose that (5) holds and, for $k \geq 0, Z_{k}$ is convex and $h_{k}$ is strictly convex.

Then, the solution of $\mathscr{P}(i, a)$ in (iv) is unique.

Assume that the hypotheses of part (iv) of the theorem are satisfied so that, for $i \geq 0, a \in X_{i}$, there is an optimal control sequence $\left\{\bar{u}_{k}(i, a)\right\}_{k \geqslant i}$, for $\mathscr{P}(i, a)$. Let $\eta_{k}: X_{k} \rightarrow R^{m}$ be defined by $\eta_{k}(x)=\bar{u}_{k}(k, x)$. Then, by the principle of optimality, $\eta_{k}$ is an optimal feedback law and an optimal feedback system is given by

$$
x_{k+1}=f_{k}\left(x_{k}, \eta_{k}\left(x_{k}\right)\right)=F_{k}\left(x_{k}\right), \quad x_{k} \in X_{k}, \quad k \geq 0 .
$$

Clearly, $F_{k}: X_{k} \rightarrow X_{k+1}$ is defined and, by (4) and nonnegativity of $h_{k}$, it follows that $\eta_{k}(0)=0$ and $F_{k}(0)=0$. We now consider the stability properties of the feedback system (25). Let $x_{k}^{*}(i, a), k \geq i$, denote the solution of (25), given $x_{i}=a \in X_{i}$. 
Theorem 4.2. Suppose that (1) satisfies Properties $C$ and $O$ and that Assumptions A1-A4 and A5 (or A5') hold. Then:

(i) for all $i \geq 0$ and $a \in X_{i}, \lim _{k \rightarrow \infty} x_{k}^{*}(i, a)=0$;

(ii) $x=0$ is the only equilibrium state of (25) and it is UAS.

The results of Theorem 4.2 can be viewed as a weak form of global stability. By removing the state-control constraints true global stability properties are obtained.

Theorem 4.3. Consider the system (25).

(i) Assume that (1) satisfies Properties C and O, $Z_{k}=R^{n} \times R^{m}, k \geq 0$, and Assumptions A2-A5 hold. Then, $x=0$ is UASL.

(ii) Assume that A6 holds. Then, $x=0$ is ES and $\exists \beta>0$ such that $\left\|\eta_{k}(a)\right\| \leq \beta\|a\|, k \geq 0, a \in R^{n}$.

Remark 4.5. The results in this section are extensions of those in Ref. 17, where we considered the special case

$$
f_{k}(x, u)=A x+B u, \quad g_{k}(x, u)=C x+D u, \quad Z_{k}=Z, \quad k \geq 0 .
$$

Theorems 4.1-4.3 require the controllability of $(A, B)$ and the observability of $(C, A)$ to ensure (through Theorems 3.1 and 3.2) that (1) satisfies Properties $\mathrm{C}$ and $\mathrm{O}$. The results in Ref. 17 are stronger in that they allow controllability to be replaced by stabilizability; also, when there are only output-control constraints, i.e.,

$$
Z=\{(x, u): y=C x+D u,(y, u) \in W\},
$$

observability can be weakened to detectability.

It is easy to see that Assumption A6 (with $q=2, p_{1}=\lambda_{1}$, and $p_{2}=2 \lambda_{2}$ ) is satisfied for the LQRP of Section 1. Thus, Theorem 4.1 implies the existence and uniqueness of the optimal feedback law and Theorem 4.3 shows that the optimal feedback system is ES.

\section{Moving-Horizon Feedback System}

We begin by completing the formulation of the $\mathrm{MH}$ feedback law described in Section 1. For $i \geq 0, a \in R^{n}$, let $\mathscr{P}\left(i, a ; M_{i}\right)$ denote the problem of minimizing the cost $\hat{J}_{i}$ in (6) subject to (1), (2), and (7). A sequence triple

$$
\left\{\left(x_{k}, y_{k}, u_{k}\right)\right\}_{k=i}^{i+M_{i}-1}
$$

is admissible for $\hat{\mathscr{P}}\left(i, a ; M_{i}\right)$ if it satisfies (1), (2) and (7). For $i \geq 0$, let

$$
\hat{X}_{i}\left(M_{i}\right)=\left\{a: \hat{\mathscr{P}}\left(i, a ; M_{i}\right) \text { has an admissible sequence }\right\} \text {, }
$$


and

$$
\hat{X}(\mathscr{M})=\bigcap_{i \geq 0} \hat{X}_{i}\left(M_{i}\right), \quad \text { where } \mathscr{M}=\left\{M_{k}\right\}_{k \geq 0} .
$$

Because $\hat{\mathscr{P}}\left(i, a ; M_{i}\right)$ is a finite-horizon optimal control problem, Assumption A2 implies that all admissible sequences for $\hat{\mathscr{P}}\left(i, a ; M_{i}\right)$ have finite costs. It is obvious that

$$
\hat{X}_{i}\left(M_{i}\right) \subset X_{i}, \quad i \geq 0, \quad \text { and } \hat{X}(M) \subset X .
$$

The following theorem concerns other properties of $\hat{X}(\mathscr{M})$ and the existence of a solution for $\hat{\mathscr{P}}\left(i, a ; M_{i}\right)$. Let us introduce the following assumption.

Assumption A7. $M_{k} \geq N_{c}, k \geq 0$, where $N_{c}$ is the index in Definition 3.2 (Property C).

Theorem 5.1. Suppose that Assumption $A 7$ is added to the hypotheses of parts (ii) and (iii) of Theorem 4.1. Then, the conclusions of Theorem 4.1 hold when $X_{i}, X$, and $\mathscr{P}(i, a)$ are replaced, respectively, by $\hat{X}_{i}\left(M_{i}\right)$, $\hat{X}(\mathcal{M})$, and $\hat{\mathscr{P}}\left(i, a ; M_{i}\right)$.

Assume that $\mathrm{A} 2$ and $\mathrm{A} 5$ (or $\mathrm{A} 5^{\prime}$ ) are satisfied, so that, for $i \geq 0$, $a \in \hat{X}_{i}\left(M_{i}\right)$, there is an optimal control sequence,

$$
\left\{\hat{u}_{k}\left(i, a ; M_{i}\right)\right\}_{k=i}^{i+M_{i}-1}
$$

for $\hat{\mathscr{P}}\left(i, a ; M_{i}\right)$ [part (iv) of Theorem 5.1]. Then, $\hat{\eta}_{k}\left(\cdot ; M_{k}\right): \hat{X}_{k}\left(M_{k}\right) \rightarrow R^{m}$, defined by $\hat{\eta}_{k}\left(x ; M_{k}\right)=\hat{u}_{k}\left(k, x ; M_{k}\right)$, is an MH feedback law. For meaningfulness, it is necessary that $f_{k}\left(x, \hat{\eta}_{k}\left(x ; M_{k}\right)\right) \in \hat{X}_{k+1}\left(M_{k+1}\right), x \in \hat{X}_{k}\left(M_{k}\right)$. Thus, the following assumption is needed.

Assumption A8. $\quad M_{k+1} \geq M_{k}-1, k \geq 0$.

The $\mathrm{MH}$ feedback system is then given by

$$
\begin{aligned}
x_{k+1} & =f_{k}\left(x_{k}, \hat{\eta}_{k}\left(x_{k} ; M_{k}\right)\right) \\
& =\hat{F}_{k}\left(x_{k} ; M_{k}\right), \quad x_{k} \in \hat{X}_{k}\left(M_{k}\right), \quad k \geq 0,
\end{aligned}
$$

where

$$
\begin{aligned}
& \hat{F}_{k}\left(\cdot ; M_{k}\right): \hat{X}_{k}\left(M_{k}\right) \rightarrow \hat{X}_{k+1}\left(M_{k+1}\right), \\
& \hat{\eta}_{k}\left(0 ; M_{k}\right)=0 \text { and } \hat{F}_{k}\left(0 ; M_{k}\right)=0 .
\end{aligned}
$$

We now consider the stability properties of the feedback system (28). Let $\hat{x}_{k}^{*}(i, a ; \mathcal{M}), k \geq i$, denote the solution of (28), given $x_{i}=a \in \hat{X}_{i}\left(M_{i}\right)$.

Theorem 5.2. Suppose that Assumptions $A 7$ and $A 8$ are added to the hypotheses of Theorem 4.2. Then, the conclusions of Theorem 4.2 hold for the feedback system (28), with $x_{k}^{*}$ and $X_{i}$ replaced, respectively, by $\hat{x}_{k}^{*}$ and $\hat{X}_{i}\left(M_{i}\right)$. 
Theorem 5.3. Suppose that Assumptions A7 and A8 are added to the hypotheses of Theorem 4.3. Then, the conclusions of Theorem 4.3 hold for the feedback system $(28)$, with $\eta_{k}(a)$ replaced by $\hat{\eta}_{k}\left(a ; M_{k}\right)$.

In Ref. 7, Kwon and Pearson consider MH feedback laws for the LQRP and treat the case where the horizons all have equal length $\left(M_{k}=M, k \geq 0\right)$. Their stability results are a special case of Theorem 5.3. The proofs in Ref. 7 utilize the linearity of the feedback law and properties of a discrete-time Riccati equation; stability of the feedback system is established in an indirect way, by demonstrating the instability of its adjoint system. Our method of proof is based on a Lyapunov theorem, with the IH cost associated with $\hat{\eta}_{k}$ serving as the Lyapunov function; linearity and the Riccati equation are not used.

\section{Convergence of the Moving-Horizon Cost}

In this section, we state results which show that, as the horizons $M_{k}$ are extended, the MH feedback law becomes a good approximation of the optimal IH feedback law.

For problem $\mathscr{P}(i, a), i \geq 0, a \in X_{i}$, let $V^{*}(i, a)$ be the optimal cost, $u_{k}^{*}(i, a)=\eta_{k}\left(x_{k}^{*}(i, a)\right)$ and $y_{k}^{*}(i, a)=g_{k}\left(x_{k}^{*}(i, a), u_{k}^{*}(i, a)\right), k \geq i$. Clearly,

$$
V^{*}(i, a)=\sum_{k=i}^{\infty} h_{k}\left(y_{k}^{*}(i, a), u_{k}^{*}(i, a)\right)
$$

Also, for problem $\hat{\mathscr{P}}\left(i, a ; M_{i}\right), i \geq 0, a \in \hat{X}_{i}\left(M_{i}\right)$, let $\hat{V}\left(i, a ; M_{i}\right)$ be the optimal cost, $\hat{u}_{k}^{*}(i, a ; \mathcal{M})=\hat{\eta}_{k}\left(\hat{x}_{k}^{*}(i, a ; \mathcal{M}) ; M_{k}\right)$ and $\hat{y}_{k}^{*}(i, a ; \mathcal{M})=$ $g_{k}\left(\hat{x}_{k}^{*}(i, a ; M), \hat{u}_{k}^{*}(i, a ; \mathcal{M})\right), k \geq i$. The IH cost associated with the MH feedback law is

$$
\hat{V}^{*}(i, a ; M)=\sum_{k=i}^{\infty} h_{k}\left(\hat{y}_{k}^{*}(i, a ; M), \hat{u}_{k}^{*}(i, a ; M)\right)
$$

It has nice properties.

Theorem 6.1. (i) Suppose that Assumptions A2, A5 (or A5'), and A8 hold, $i \geq 0$ and $a \in \hat{X}_{i}\left(M_{i}\right)$. Then, $V^{*}(i, a), \hat{V}\left(i, a ; M_{i}\right)$ and $\hat{V}^{*}(i, a ; \mathcal{M})$ exist and satisfy

$$
V^{*}(i, a) \leq \hat{V}^{*}(i, a ; \mathcal{M}) \leq \hat{V}\left(i, a ; M_{i}\right) .
$$

(ii) Suppose that the hypotheses of Theorem 4.2 are satisfied. Then, given $i \geq 0, a \in X_{i}$, and $\delta>0, \exists M(i, a, \delta) \geq N_{c}$ such that

$$
M_{i} \geq M(i, a, \delta) \Rightarrow a \in \hat{X}_{i}\left(M_{i}\right) \quad \text { and } \quad \hat{V}\left(i, a ; M_{i}\right) \leq V^{*}(i, a)+\delta \text {. }
$$


Moreover, $\exists r>0$ and, given $\delta>0, \exists$ a positive integer $M(\delta) \geq N_{c}$ such that

$$
\hat{V}\left(i, a ; M_{i}\right) \leq V^{*}(i, a)+\delta, \quad i \geq 0, \quad M_{i} \geq M, \quad a \in N(r) .
$$

The lower bound in (31) is obvious from the optimality for $\mathscr{P}(i, a)$. However, the upper bound needs a more detailed proof. Implicit in (33) is the conclusion that $N(r) \subset \hat{X}_{i}(M), i \geq 0$, a fact which is obvious from part (ii) of Theorem 5.1 .

Remark 6.1. Parts (i) and (ii) can be combined to describe the convergence of $\hat{V}^{*}(i, a ; \mathcal{M})$ to $V^{*}(i, a)$. Note that the bounds on $\hat{V}\left(i, a ; M_{i}\right)$ and $\hat{V}^{*}(i, a ; M)$ depend only on $M_{i} \in \mathcal{M}$; the remaining elements of $M$ are only required to satisfy Assumption A8. Clearly, part (ii) implies Assumption A7.

In part (ii) of Theorem 6.1, (33) is stronger than (32) in that the convergence is uniform with respect to $i$ and $a$; it is weaker in that it applies only locally. Under stronger hypotheses stronger convergence properties can be obtained.

Theorem 6.2. (i) If the hypotheses of part (i) of Theorem 4.3 hold, then given any $r>0$ and $\delta>0 \exists$ a positive integer $M(r, \delta)$ such that (33) holds.

(ii) Assumption A6 implies that, given any $\delta>0, \exists$ a positive integer $M(\delta)$ such that

$$
\hat{V}\left(i, a ; M_{i}\right) \leq(1+\delta) V^{*}(i, a), \quad i \geq 0, \quad M_{i} \geq M, \quad a \in R^{n} .
$$

Part (ii) of Theorem 6.2, specialized to the LQRP, was obtained by Kwon and Pearson (Ref. 7).

\section{Proofs}

We now prove the results in Sections 4-6. Most of the details required for proving Theorems 4.1-4.3 are omitted, since they parallel those which we use in proving Theorems 5.1-5.3. nonempty.

Proof of Theorem 5.1. By (4), $0 \in \hat{X}_{i}\left(M_{i}\right)$; thus, $\hat{X}_{i}\left(M_{i}\right), i \geq 0$, are

Now, consider part (ii). By Assumption A1, $\exists \epsilon>0$ such that $N(\epsilon) \subset Z$. Choose $\lambda>0$ such that $W_{c}(\lambda) \leq \epsilon$, where $W_{c}$ is the $\mathscr{H}_{\infty}$ function in Definition 3.2. Given $i \geq 0, a \in R^{n}$, let $\left\{\left(x_{k}, y_{k}, u_{k}\right)\right\}_{k \geq i}$ be the sequence given by Definition 3.2 with $y_{k}=g_{k}\left(x_{k}, u_{k}\right)$. If $\|a\| \leq \lambda$, it follows by Assumptions 
A1, A7 and (14) that $\left\{\left(x_{k}, y_{k}, u_{k}\right)\right\}_{k=1}^{i+M_{i}-1}$ is admissible for $\hat{\mathscr{P}}\left(i, a ; M_{i}\right)$. Since $\lambda$ is independent of $i, N(\lambda) \subset \hat{X}(\mathcal{M})$ which proves part (ii).

Part (iii) follows immediately, since $Z=R^{n+m}$ implies that $\epsilon$, and hence $\lambda$, can be arbitrarily large.

Part (iv) is proved using Theorem 10.1. Define

$$
\begin{aligned}
& W_{k}=Z_{k}, \quad i \leq k \leq i+M_{i}-1, \\
& W_{k}=\{(0,0)\}, \quad k \geq M_{i} ; \\
& T_{i}=\{a\} ; \\
& \mathscr{L}_{k}(x, u)=h_{k}\left(g_{k}(x, u), u\right), \quad k \geq i, \quad(x, u) \in R^{n+m} .
\end{aligned}
$$

Clearly, Assumptions A2 and A5 imply conditions (a)-(c) of Theorem 10.1. Then, define

$$
\phi_{k}(u)=H_{2}(\|u\|), \quad k \geq i, \quad u \in R^{m},
$$

to see that Assumptions A5 implies condition (d). If $a \in \hat{X}_{i}\left(M_{i}\right)$, condition (e) is also satisfied. Thus, by Theorem $10.1, \hat{\mathscr{P}}\left(i, a ; M_{i}\right)$ has a solution for all $i \geq 0, a \in \hat{X}_{i}\left(M_{i}\right)$. If Assumption $\mathrm{A} 5$ is replaced by $\mathrm{A} 5$, then the existence result still holds since condition (b) of A $5^{\prime}$ implies the nonnegativity of $h_{k}$, and condition (c) of $A 5^{\prime}$ implies condition $\left(d_{1}\right)$ of 'Theorem 10.1 .

Now, consider part $(v)$. Suppose that

$$
\left\{\left(x_{k}^{1}, y_{k}^{1}, u_{k}^{1}\right)\right\}_{k=i}^{i+M_{i}-1}, \quad\left\{\left(x_{k}^{2}, y_{k}^{2}, u_{k}^{2}\right)\right\}_{k=i}^{i+M_{i}-1}
$$

are two distinct solutions of $\hat{\mathscr{P}}\left(i, a ; M_{i}\right)$. Thus, $\exists j: i \leq j \leq i+M_{i}-1$ and $u_{j}^{1} \neq u_{j}^{2}$. Let $\mu \in(0,1)$ and

$$
\begin{aligned}
\left(x_{k}, y_{k}, u_{k}\right) & =\mu\left(x_{k}^{1}, y_{k}^{1}, u_{k}^{1}\right) \\
& +(1-\mu)\left(x_{k}^{2}, y_{k}^{2}, u_{k}^{2}\right), \quad i \leq k \leq i+M_{i}-1 .
\end{aligned}
$$

By (5) and convexity of $Z_{k},\left\{\left(x_{k}, y_{k}, u_{k}\right)\right\}_{k=i}^{i+M_{i}-1}$ is admissible to $\hat{\mathscr{P}}\left(i, a ; M_{i}\right)$. By convexity of $h_{k}$,

$$
h_{k}\left(y_{k}, u_{k}\right) \leq \mu h_{k}\left(y_{k}^{1}, u_{k}^{1}\right)+(1-\mu) h_{k}\left(y_{k}^{2}, u_{k}^{2}\right), \quad i \leq k \leq i+M_{i}-1 ;
$$

also, by the strict convexity of $h_{j}$,

$$
h_{j}\left(y_{j}, u_{j}\right)<\mu h_{j}\left(y_{j}^{1}, u_{j}^{1}\right)+(1-\mu) h_{j}\left(y_{j}^{2}, u_{j}^{2}\right) .
$$

Thus,

$$
\sum_{k=i}^{i+M_{i}-1} h_{k}\left(y_{k}, u_{k}\right)<\hat{V}\left(i, a ; M_{i}\right)
$$

which is a contradiction. 
Proof of Theorem 4.1. It is essentially the same as the proof of Theorem 5.1, except that $\mathscr{P}(i, a)$ replaces $\hat{\mathscr{P}}\left(i, a ; M_{i}\right), X_{i}$ replaces $\hat{X}_{i}\left(M_{i}\right)$, and $X$ replaces $\hat{X}(\mathscr{M})$.

Stability properties of the IH and MH systems will be established by using the following theorem, which is mainly an extension of a Lyapunov stability theorem (Refs. 14 and 15) to systems with a constrained state space. We omit its proof, since it is similar to the proofs in Refs. 14 and 15.

Theorem 7.1. Consider the system (9) and the associated notations immediately below (9). Assume that $0 \in X_{k}, F_{k}(0)=0, k \geq 0$. Suppose that $\exists V: Y \rightarrow R, \alpha \in \mathscr{K}_{\infty}, \beta \in \mathscr{K}_{\infty}, \gamma \in \mathscr{H}_{\infty}, \lambda>0$ and a positive integer $L$ which satisfy the following conditions:

(a) $V(i, a) \leq \beta(\|a\|), \quad \forall(i, a) \in Y, a \in N(\lambda)$;

(b) $V(i, a) \geq \alpha(\|a\|), V(i, a)-V\left(i+L, x_{i+L}^{*}(i, a)\right) \geq y(\|a\|)$, $\left.V(i, a)-V(i+1), x_{i+1}^{*}(i, a)\right) \geq 0, \quad \forall(i, a) \in Y$.

Then:

(i) $\quad x=0$ is UAS;

(ii) if $N(\lambda)$ is replaced by $R^{n}, x=0$ is UASL;

(iii) if $N(\lambda)$ is replaced by $R^{n}$ and $\exists$ positive numbers $\alpha_{1}, \beta_{1}, \gamma_{1}$, and $q$ such that $\alpha(s)=\alpha_{1} s^{q}, \beta(s)=\beta_{1} s^{q}, \gamma(s)=\gamma_{1} s^{q}$, the origin is ES and the degree of ES is at least $\left(\left(\beta_{1}-\gamma_{1}\right) / \beta_{1}\right)^{1 / q L}$.

The functions $V^{*}$ and $\hat{V}^{*}$ introduced in Section 6 will serve as the Lyapunov functions, for the $\mathrm{IH}$ and $\mathrm{MH}$ feedback systems respectively. The bounds in condition (b) of Theorem 7.1 will be established using Property $\mathrm{O}$ and Assumption A5; condition (a) will be based on Property $\mathrm{C}$, Assumptions A3, A4, A7, and the following lemma.

Lemma 7.1. For $i \geq 0, a \in R^{n}$, let $\tilde{V}(i, a)$ be defined by

$$
\tilde{V}(i, a)=\sum_{k=i}^{i+N_{c}-1} h_{k}\left(y_{k}, u_{k}\right)=\sum_{k=i}^{\infty} h_{k}\left(y_{k}, u_{k}\right)
$$

where $\left\{\left(y_{k}, u_{k}\right)\right\}_{k \gg i}$ is the sequence defined in the proof of part (ii) of Theorem 5.1. Then:

(i) Assumptions A3 and A4 imply the existence of a $\mathscr{T}_{\infty}$ function $\tilde{\varphi}$ such that

$$
\tilde{V}(i, a) \leq \tilde{\varphi}(\|a\|), \quad i \geq 0, a \in R^{n} ;
$$

(ii) Assumption A6 implies (36) with

$$
\tilde{\varphi}(s)=\tilde{p} s^{q},
$$

where $\tilde{p}>0$ and $q>0$ is the constant in A6. 
Proof. For $i \geq 0, a \in R^{n}$, Assumptions A3 and (14) yield

$$
\left\|\left(y_{k}, u_{k}\right)\right\| \leq G\left(W_{c}(\|a\|)\right)+W_{c}(\|a\|)=\tilde{W}(\|a\|), \quad k \geq i .
$$

By this and A4, we get the bound

$$
\tilde{V}(i, a) \leq \sum_{k=i}^{i+N_{k}-1} H_{1}\left(\left\|\left(y_{k}, u_{k}\right)\right\|\right) \leq \tilde{\varphi}(\|a\|)
$$

where

$$
\tilde{\varphi}(s)=N_{c} H_{1}(\tilde{W}(s)) \text {. }
$$

Clearly, $\tilde{\varphi} \in \mathscr{K}_{\infty}$. This proves part (i). By Theorem 3.1 and A6, $W_{c}(s)=p_{c} s$ and $H_{1}(s)=p_{2} s^{q}$. Also,

$$
G(s)=d s, \quad \text { where } d=\sup _{k \geq 0}\left\|\left[C_{k} D_{k}\right]\right\| .
$$

These results imply that $\tilde{\varphi}$ has the form of part (ii).

Proof of Theorem 5.2. We begin by developing an inequality relating the functions $\hat{V}$ and $\hat{V}^{*}$ defined in Section 6. Define

$$
\hat{Y}=\left\{(i, a): i \geq 0, a \in \hat{X}_{i}\left(M_{i}\right)\right\} \text {. }
$$

In what follows, we use $\hat{x}_{k}^{*}, \hat{y}_{k}^{*}$, and $\hat{u}_{k}^{*}$ as abbreviations for $\hat{x}_{k}^{*}(i, a ; \mathcal{M})$, $\hat{y}_{k}^{*}(i, a ; \mathcal{M})$, and $\hat{u}_{k}^{*}(i, a ; \mathcal{M})$. For $(i, a) \in \hat{Y}$ and $k \geq i$,

$$
\hat{V}\left(k, \hat{x}_{k}^{*} ; M_{k}\right)=h_{k}\left(\hat{y}_{k}^{*}, \hat{u}_{k}^{*}\right)+c^{*},
$$

where $c^{*}$ is the optimal cost for $\hat{\mathscr{P}}\left(k+1, \hat{x}_{k+1}^{*} ; M_{k}-1\right)$. By A8 and the optimality for $\hat{\mathscr{P}}\left(k+1, \hat{x}_{k+1}^{*} ; M_{k+1}\right)$,

$$
\hat{V}\left(k, \hat{x}_{k}^{*} ; M_{k}\right)-\hat{V}\left(k+1, \hat{x}_{k+1}^{*} ; M_{k+1}\right) \geq h_{k}\left(\hat{y}_{k}^{*}, \hat{u}_{k}^{*}\right) .
$$

Successive use of this inequality yields

$$
\hat{V}\left(i, a ; M_{i}\right) \geq \hat{V}\left(i, a ; M_{i}\right)-\hat{V}\left(j, \hat{x}_{j}^{*} ; M_{j}\right) \geq \sum_{k=i}^{j-1} h_{k}\left(\hat{y}_{k}^{*}, \hat{u}_{k}^{*}\right), \quad j>i .
$$

Since $j$ can be arbitrarily large,

$$
\hat{V}\left(i, a ; M_{i}\right) \geq \sum_{k=i}^{\infty} h_{k}\left(\hat{y}_{k}^{*}, \hat{u}_{k}^{*}\right)=\hat{V}^{*}(i, a ; \mathcal{M})
$$

First, consider the proof for the case where A5 holds. Let $(i, a) \in \hat{Y}$. Since

$$
\hat{V}^{*}(i, a ; \mathcal{M}) \leq \hat{V}\left(i, a ; M_{i}\right)<\infty,
$$

A5 implies that

$$
H_{2}\left(\left\|\left(\hat{y}_{k}^{*}, \hat{u}_{k}^{*}\right)\right\|\right) \rightarrow 0, \quad \text { as } k \rightarrow \infty .
$$


$H_{2} \in \mathscr{K}_{\infty}$ implies $\left\|\left(\hat{y}_{k}^{*}, \hat{u}_{k}^{*}\right)\right\| \rightarrow 0$, as $k \rightarrow \infty$. It follows from Property $O$ that $W_{0}\left(\left\|\hat{x}_{k}^{*}\right\|\right) \rightarrow 0$ as $k \rightarrow \infty$. Since $W_{0} \in \mathscr{K}_{\infty},\left\|\hat{x}_{k}^{*}\right\| \rightarrow 0$ as $k \rightarrow \infty$, which proves part (i).

By part (i), the only equilibrium point for (28) is $x=0$. To prove UAS of $x=0$, we use part (i) of Theorem 7.1 with $\hat{V}^{*}$ serving as the Lyapunov function. Let $i \geq 0$ and $a \in N(\lambda)$, where $\lambda>0$ is the constant defined in the proof of Theorem 5.1. By A7 and the definition of $\lambda$, the sequence $\left\{\left(x_{k}, y_{k}, u_{k}\right)\right\}_{k=i}^{i+M_{i}-1}$ used in the proof of part (ii) of Theorem 5.1 is admissible for $\hat{\mathscr{P}}\left(i, a ; M_{i}\right)$. Optimality for $\hat{\mathscr{P}}\left(i, a ; M_{i}\right)$ together with (36) and (38) yields

$$
\begin{aligned}
\hat{V}^{*}(i, a ; M) & \leq \hat{V}\left(i ; a ; M_{i}\right) \\
& \leq \tilde{\varphi}(\|a\|), \quad(i, a) \in \hat{Y}, \quad a \in N(\lambda) .
\end{aligned}
$$

Let $(i, a) \in \hat{Y}$. Then Assumption $A 5, H_{2} \in \mathscr{K}_{\infty}$, and Property $\mathrm{O}$ imply that

$$
\begin{aligned}
\sum_{k=i}^{i+N_{0}-1} h_{k}\left(\hat{y}_{k}^{*}, \hat{u}_{k}^{*}\right) & \geq H_{2}\left(\max _{i \leq k \leq i+N_{0}-1}\left\|\left(\hat{y}_{k}^{*}, \hat{u}_{k}^{*}\right)\right\|\right) \\
& \geq H_{2}\left(\frac{1}{N_{0}} \sum_{k=i}^{i+N_{0}-1}\left\|\left(\hat{y}_{k}^{*}, \hat{u}_{k}^{*}\right)\right\|\right) \geq \bar{\varphi}(\|a\|),
\end{aligned}
$$

where $\bar{\varphi}: R_{+} \rightarrow R_{+}$, defined by

$$
\bar{\varphi}(s)=H_{2}\left(\frac{1}{N_{0}} W_{0}(s)\right),
$$

is in $\mathscr{K}_{\infty}$, because $H_{2}$ and $W_{0}$ are in $\mathscr{K}_{\infty}$. Thus,

$$
\hat{V}^{*}(i, a ; \mathcal{M}) \geq \sum_{k=i}^{i+N_{0}-1} h_{k}\left(\hat{y}_{k}^{*}, \hat{u}_{k}^{*}\right) \geq \bar{\varphi}(\|a\|), \quad(i, a) \in \hat{Y} .
$$

Moreover,

$$
\begin{aligned}
& \hat{V}^{*}(i, a ; \mathcal{M})-\hat{V}^{*}\left(i+N_{0}, \hat{x}_{i+N_{0}}^{*} ; \mathcal{M}\right) \\
& =\sum_{k=i}^{i+N_{0}-1} h_{k}\left(\hat{y}_{k}^{*}, \hat{u}_{k}^{*}\right) \geq \bar{\varphi}(\|a\|), \quad(i, a) \in \hat{Y},
\end{aligned}
$$

and by the nonnegativity of $h_{k}$,

$$
\begin{aligned}
& \hat{V}^{*}(i, a ; M)-\hat{V}^{*}\left(i+1, \hat{x}_{i+1}^{*} ; \mathcal{M}\right) \\
& =h_{i}\left(\hat{y}_{i}^{*}, \hat{u}_{i}^{*}\right) \geq 0, \quad(i, a) \in \hat{Y} .
\end{aligned}
$$

With $\alpha=\gamma=\bar{\varphi}, \beta=\tilde{\varphi}, V=\hat{V}^{*}, Y=\hat{Y}$, and $L=N_{0}$, it is seen that conditions (a) and (b) of Theorem 7.1 hold. Thus, the proof of part (ii) is complete. 
Now, consider the replacement of Assumption A5 by A5'. Assumption A5 is used in the proofs to prove part (i) and derive (42)-(44). The proof of part (i) is obvious from (a) and (b) of $A 5^{\prime}$. Conditions (a) and (b) also imply that

$$
\begin{aligned}
\hat{V}^{*}(i, a ; \mathcal{M}) & \geq \hat{V}^{*}(i, a ; \mathcal{M})-\hat{V}^{*}\left(i+1, \hat{x}_{i+1}^{*} ; \mathcal{M}\right) \geq h_{i}\left(\hat{y}_{i}^{*}, \hat{u}_{i}^{*}\right) \\
& \geq H_{3}\left(\left\|\hat{y}_{i}^{*}\right\|\right) \geq H_{3}(\|a\|) \geq 0, \quad(i, a) \in \hat{Y},
\end{aligned}
$$

This implies (42)-(44), with $\bar{\varphi}(s)=H_{3}(s)$ and $N_{0}=1$.

Proof of Theorem 5.3. The proof of part (i) is omitted, since by part (ii) of Theorem 7.1 it is an obvious modification of the proof of part (ii) of Theorem 5.2. Part (ii) is established as follows. By Assumption A6 and Theorem 3.2,

$$
H_{2}(s)=p_{1} s^{q}, \quad W_{0}(s)=p_{0} s .
$$

Thus, $\bar{\varphi}$ in (41) becomes

$$
\bar{\varphi}(s)=\bar{p} s^{q},
$$

where $\bar{p}=p_{1}\left(p_{0} / N_{0}\right)^{q}$. This together with (37), (39), (42)-(44), and part (iii) of Theorem 7.1 proves ES. The required bound on $\hat{\eta}_{k}\left(\cdot ; M_{k}\right)$ is easy to establish by using (22), (36), (37), (39), and letting $\beta=\left(\tilde{p} / p_{1}\right)^{1 / q}$.

Proofs of Theorems 4.2-4.3. The proofs are obtained from those of Theorems $5.2-5.3$ by:

(i) omitting $M_{i}$ and $\mathscr{M}$ from all the notations;

(ii) omitting all the hats;

(iii) defining $V(i, a)=V^{*}(i, a)$, for $i \geq 0, a \in X_{i}$.

Because of (iii), the inequality corresponding to (38) is immediate and eliminates the need for Assumption A8. Also, the relation parallel to (39) follows from Lemma 7.1 and the optimality for $\mathscr{P}(i, a)$; thus Assumption A7 is also not needed.

Remark 7.1. It may seem surprising that simply removing the statecontrol constraints changes UAS [part (ii) of Theorems 4.2 and 5.2] to UASL [part (i) of Theorems 4.3 and 5.3]. The change hinges on the fact that, with constraints, (39) holds only locally: the sequence triple $\left\{x_{k}, y_{k}, u_{k}\right\}_{k=i}^{i+M_{i}-1}$, which is used in deriving (39), may not be admissible to $\hat{\mathscr{P}}\left(i, a ; M_{i}\right)$, for all $i \geq 0$ and $a \in \hat{X}_{i}\left(M_{i}\right)$. If $(39)$ can be made global (i.e. $\lambda=\infty$ ) by some other means, then part (ii) of Theorem 5.2 [4.2] and part (i) of Theorem 5.3 [4.3] merge to yield UASL. 
Proof of Theorem 6.1. The proof of part (i) follows directly from part (iv) of Theorems 4.1 and 5.1, Assumption A8, (38), and the optimality for $\mathscr{P}(i, a)$. Consider the proof of (32). Let $i \geq 0, a \in X_{i}$ be given. Let

$$
M \geq N_{c}, \quad K=M-N_{c},
$$

and define the sequence triple $\left\{\left(\bar{x}_{k}, \bar{y}_{k}, \bar{u}_{k}\right)\right\}_{k \geq i}$ by

$$
\begin{array}{lll}
\left(\bar{x}_{k}, \bar{y}_{k}, \bar{u}_{k}\right)=\left(x_{k}^{*}(i, a), y_{k}^{*}(i, a), u_{k}^{*}(i, a)\right), & & i \leq k \leq i+K-1, \\
\left(\bar{x}_{k}, \bar{y}_{k}, \bar{u}_{k}\right)=\left(\tilde{x}_{k}, \tilde{y}_{k}, \tilde{u}_{k}\right), & & k \geq i+K,
\end{array}
$$

where $\tilde{y}_{k}=g_{k}\left(\tilde{x}_{k}, \tilde{u}_{k}\right)$ and $\left\{\left(\tilde{x}_{k}, \tilde{u}_{k}\right)\right\}_{k \geq i+K}$ is the sequence in Definition 3.2 corresponding to the initial values

$$
(i, a): i=i+K, \quad \tilde{a}=x_{i+K}^{*}(i, a) .
$$

By (36), $\bar{V}(i, a ; M)$, the cost given by the sequence triple $\left\{\left(\bar{x}_{k}, \bar{y}_{k}, \bar{u}_{k}\right)\right\}_{k \geq i}$ to $J_{i}$ in (3), satisfies

$$
\bar{V}(i, a ; M) \leq V^{*}(i, a)+\tilde{\varphi}\left(\left\|x_{i+K}^{*}(i, a)\right\|\right) .
$$

Now, consider the selection of $M(i, a, \delta)$. By part (i) of Theorem 4.2 , $\left\|x_{i+K}^{*}(i, a)\right\| \rightarrow 0$, as $K \rightarrow \infty$. Choose $K(i, a, \delta)$ such that $\dot{\varphi}\left(\left\|x_{i+K}^{*}(i, a)\right\|\right) \leq \delta$ and $\left\|x_{i+K}^{*}(i, a)\right\| \leq \lambda$, where $\lambda$ is the constant in the proof of Theorem 5.1; then, let $M(i, a, \delta)=K(i, a, \delta)+N_{c}$. By the definition of $\lambda$, $\left\{\left(\bar{x}_{k}, \bar{y}_{k}, \bar{u}_{k}\right)\right\}_{k=i}^{i+M-1}$ is admissible for $\hat{\mathscr{P}}(i, a ; M)$. So, $a \in \hat{X}_{i}(M(i, a, \delta))$; also, by (46), the optimality for $\hat{\mathscr{P}}(i, a ; M)$, and the definition of $K$,

$$
\hat{V}(i, a ; M) \leq \bar{V}(i, a ; M) \leq V^{*}(i, a)+\delta .
$$

Implication (32) follows immediately from $\hat{X}_{i}(M) \subset \hat{X}_{i}\left(M_{i}\right)$ and the optimality for $\hat{\mathscr{P}}\left(i, a ; M_{i}\right)$.

Consider (33). Given $\delta>0$, choose $\sigma>0$ such that $\tilde{\varphi}(\sigma) \leq \delta$ and $\sigma \leq \lambda$, where $\lambda$ is the constant defined in the proof of Theorem 5.1. By part (ii) of Theorem 4.2, $\exists T(\sigma)>0$ such that (10) holds. Define

$$
K(\delta)=T \text { and } M(\delta)=K+N_{0} .
$$

Now, let $i \geq 0$ and $a \in N(r)$, where $r>0$ is the constant in (10). Then, (10) implies that

$$
\left\|x_{i+K}^{*}(i, a)\right\| \leq \sigma \leq \lambda .
$$

By the definition of $\lambda,\left\{\left(\bar{x}_{k}, \bar{y}_{k}, \bar{u}_{k}\right)\right\}_{k=i}^{i+M-1}$, the sequence triple defined while establishing (32), is admissible for $\hat{\mathscr{P}}(i, a ; M)$. Thus, we get (47) from (46) the definition of $\sigma, K, M$, and the optimality for $\hat{\mathscr{P}}(i, a ; M)$. Relation (33) follows immediately from the optimality for $\hat{\mathscr{P}}\left(i, a ; M_{i}\right)$. 
Proof of Theorem 6.2. The proof of part (i) is identical to that of (33) if in the proof we use part (i) of Theorem 4.3 instead of part (ii) of Theorem 4.2. To prove part (ii), let $\delta>0$ be given. Choose a positive integer $K$ such that $\delta \geq \tilde{p}\left(\phi \zeta^{\bar{K}}\right)^{q} / \bar{p}$, where $\phi, \zeta, q, \tilde{p}$, and $\bar{p}$ are the constants in $(11),(22)$, (37), and (45). This is possible, since $0 \leq \zeta<1$. Let $M=K+N_{c}$. Corresponding to (42), we have

$$
V^{*}(i, a) \geq \bar{\varphi}(\|a\|), \quad i \geq 0, \quad a \in X_{i} .
$$

By this, (38), (46), (37), (11), and (45), we have, for $i \geq 0, a \in R^{n}, M_{i} \geq M$,

$$
\begin{aligned}
\hat{V}\left(i, a ; M_{i}\right) & \leq \bar{V}(i, a ; M) \leq V^{*}(i, a)+\tilde{p}\left\|x_{i+K}^{*}(i, a)\right\|^{q} \\
& \leq V^{*}(i, a)+\tilde{p}\left(\phi \zeta^{K}\right)^{q}\|a\|^{q} \\
& \leq V^{*}(i, a)+\delta \bar{p}\|a\|^{q} \leq(1+\delta) V^{*}(i, a) .
\end{aligned}
$$

\section{Illustrative Examples}

The general problem formulation (1)-(3) offers a much richer approach to feedback design than the LQRP. In this section, we illustrate some of the possible advantages of the formulation by considering two examples.

Example 8.1. The example is based on a crane which has been considered in Ref. 18 and elsewhere. It consists of an overhead trolley which runs on straight frictionless rails with its load suspended on an inextensible cable from its center of gravity. The objective is to move the trolley from a given position to a reference point with the load starting and finishing in a stationary position directly beneath the trolley. Discretizing the continuous-time model in Ref. 18 by using a zero-order hold (with period $T=0.3$ ) gives the following difference equations:

$$
\begin{aligned}
& x_{k+1}^{1}=x_{k}^{1}+0.3 x_{k}^{2}+0.036 x_{k}^{3}+0.0036 x_{k}^{4}+0.08 u_{k}, \\
& x_{k+1}^{2}=x_{k}^{2}+0.234 x_{k}^{3}+0.036 x_{k}^{4}+0.534 u_{k}, \\
& x_{k+1}^{3}=0.92 x_{k}^{3}+0.292 x_{k}^{4}-0.08 u_{k}, \\
& x_{k+1}^{4}=-0.526 x_{k}^{3}+0.92 x_{k}^{4}-0.526 u_{k} .
\end{aligned}
$$

Here, $x_{k}^{1}, x_{k}^{2}, x_{k}^{3}$, and $x_{k}^{4}$ denote, respectively, the position of the trolley (from the reference point), its velocity, the angle of the cable from the vertical, and its angular velocity at time $k T$. The control $u_{k}$ is the normalized 
horizontal force applied on the trolley in the interval $k T \leq t \leq(k+1) T$. Therefore, it satisfies the constraint $\left|u_{k}\right| \leq 1$.

Let $l=n=4$ and $y_{k}=x_{k}$, where $x_{k}=\left(x_{k}^{1}, x_{k}^{2}, x_{k}^{3}, x_{k}^{4}\right)$. We consider $l_{1}$ and $l_{2}$ cost functions:

$$
h^{1}(y, u)=|y|+\rho|u| \text { and } h^{2}(y, u)=\|y\|^{2}+(\rho u)^{2},
$$

where $|y|$ denotes the $l_{1}$ norm of the vector $y$ and $\rho=10^{-3}$. The following abbreviations will be used: $\mathrm{UC}=$ unconstrained, $\mathrm{CC}=$ control constrained. Our first computation concerns the FOMP implementation for the UC-MH$l_{1}$ problem $\left(Z=R^{n+m}, h_{k}=h^{1}, M_{k}=M\right)$. An efficient $l_{1}$ computer code, based on linear programming (Ref. 19), was used to solve the finitedimensional $\mathrm{MH}$ problems at each time step. Continuous-time responses $x^{1}(t)$ and $x^{3}(t)$, corresponding to $x_{0}=(-5,0,0,0)$, are given in Fig. 1 for $M=10,15,20$, and 30 . There is no change in the solutions for $M \geq 20$. It is believed, but has not been proved, that for $M \geq 20$ solutions for the UC-MH- $I_{1}$ and UC-IH- $I_{1}$ problems are the same, with the response deadbeat in 20 steps.

The UC-IH- $l_{2}$ problem is a time-invariant LQRP and so its optimal control law is given by $u_{k}=K x_{k}$, where $K$ is obtained by solving a discretetime algebraic Riccati equation. The largest control magnitudes for the UC-MH- $l_{1}(M=30)$ and UC-IH- $l_{2}$ solutions (respectively, 7.67 and 4.56) are considerably higher than the desired limit of 1.0. Adding the control constraint $\left|u_{k}\right| \leq 1$ gives the CC-MH- $l_{1}$ and CC-MH- $l_{2}$ problems. Again, it is believed that CC-MH- $l_{1}(M \geq 30)$ and $\mathrm{CC}-\mathrm{IH}-l_{1}$ solutions are identical. An efficient quadratic programming code (Ref. 20) was used to obtain the FOMP implementations for the CC-MH- $l_{2}$ problems. The responses $x^{l}(t)$ and $x^{3}(t)$ corresponding to the UC-MH- $l_{1}(M=30)$, UC-IH- $l_{2}, \mathrm{CC}-\mathrm{MH}-l_{1}$ $(M=30)$, and CC-MH- $l_{2}(M=30)$ problems are shown in Fig. 2. Some useful indicators of solution properties are given in Table 1.

The following observations can be made. Apart from keeping the force within limits, the control constraint also causes the magnitudes of the velocities to be considerably reduced, without much loss in the overall speed of response. Also, the IH costs only increase about $10 \%$ despite the very large reduction in control amplitude. It is also our general experience that good response properties are obtained when the control term is removed from the cost function (i.e. $\rho=0$ ) and, instead, a compactness constraint is placed on the control. By Assumption A5 ${ }^{\prime}$ and Theorems 4.2 and 5.2, this problem has a sound theoretical basis. The $l_{1}$ cost offers a deadbeat solution even when the control constraint is imposed. Also, the maximum values in Table 1 for the CC-MH- $l_{1}$ and CC-MH- $l_{2}$ solutions are comparable. Therefore, with the control constraint, the $l_{1}$ cost yields a better quality of regulation compared to the $l_{2}$ cost. 

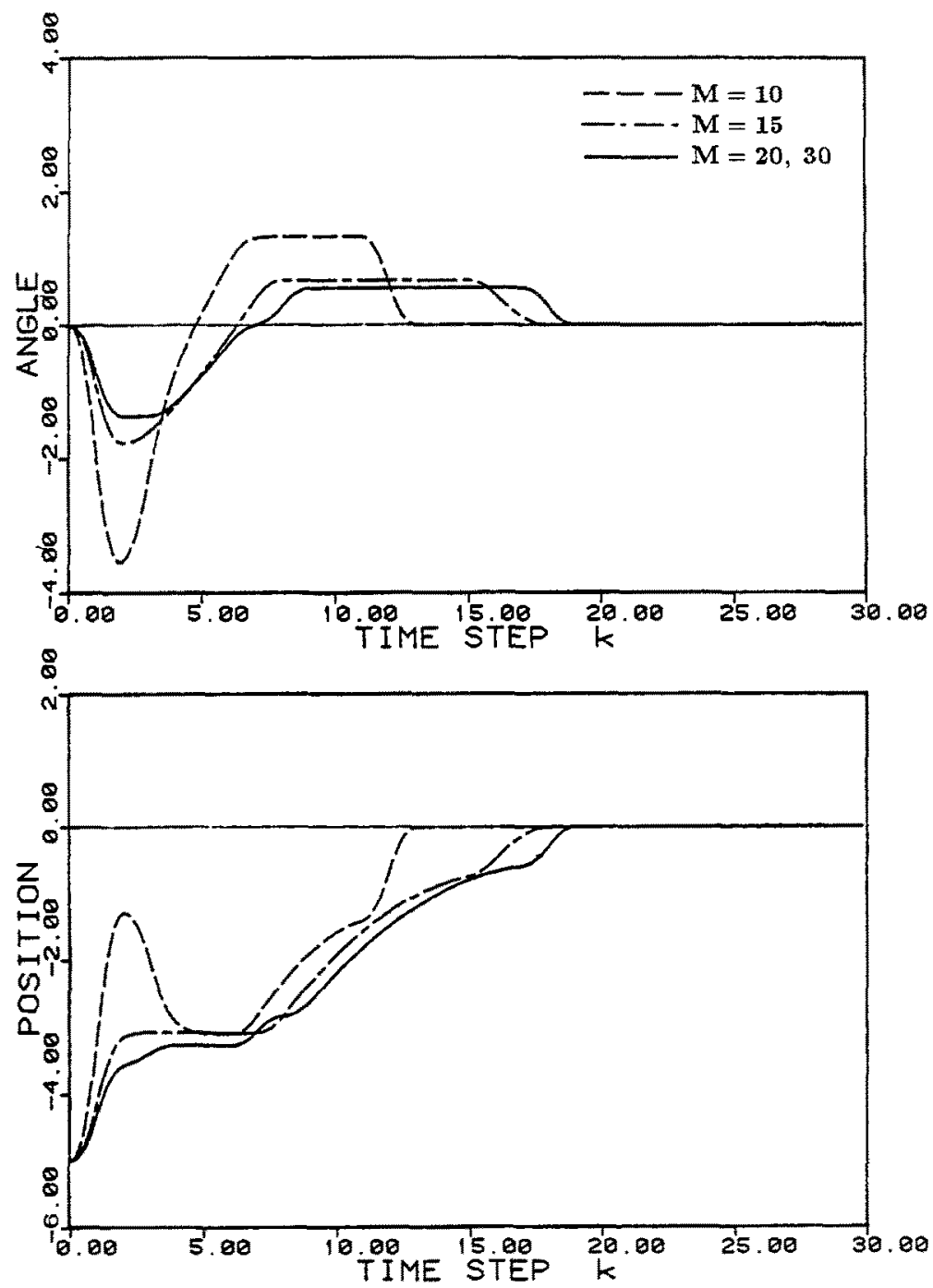

Fig. 1. Responses for the UC-MH-l $l_{1}$ problems.

Example 8.2. This example is the double integrator

$$
\dot{x}^{1}(t)=x^{2}(t), \quad \dot{x}^{2}(t)=u(t),
$$

with control constraint $|u| \leq 1$. We consider two approaches to the quadratic regulation problem. The first is a commonly used pragmatic approach: find a linear feedback law by solving the LQRP and then add an appropriate 

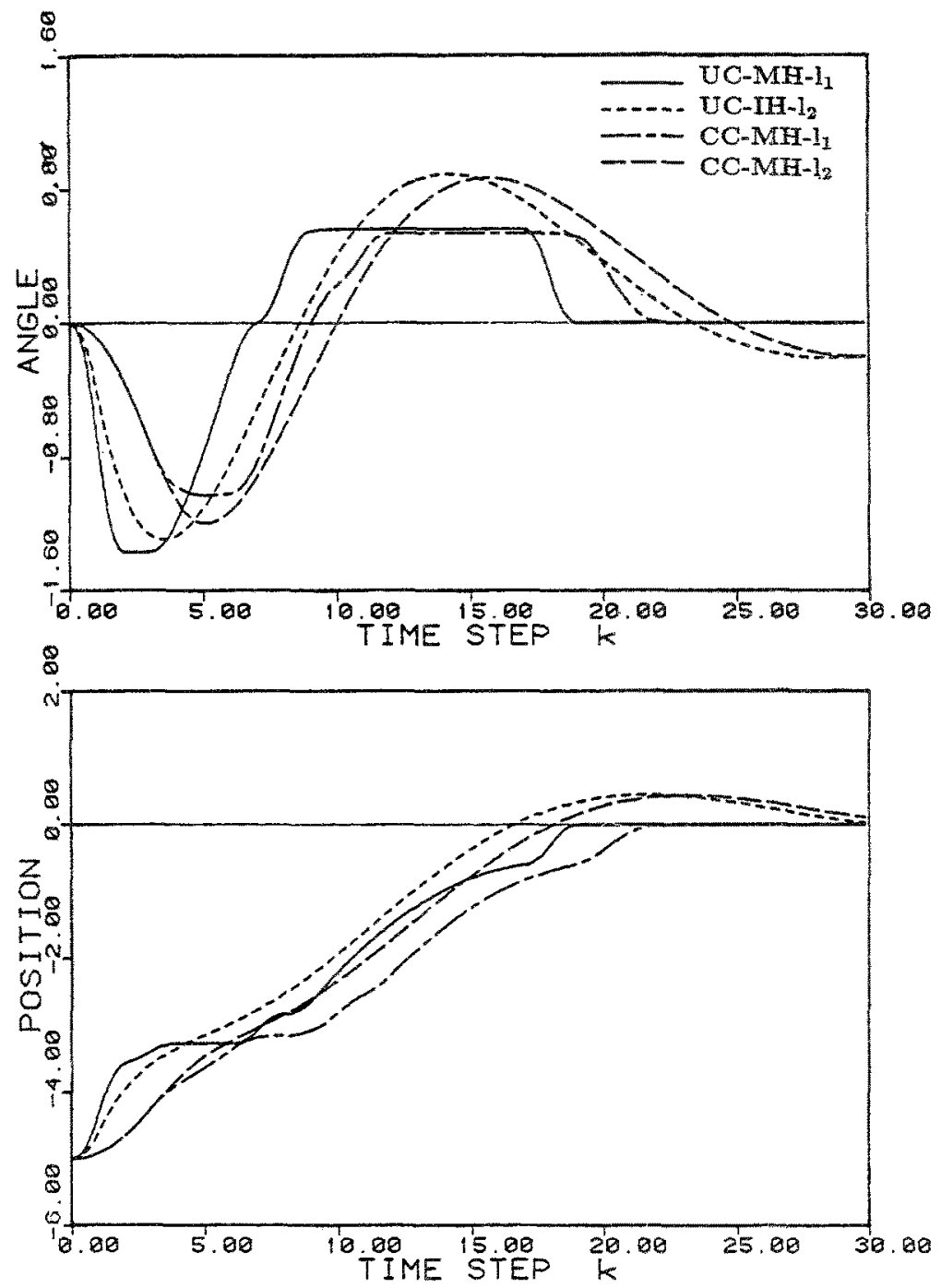

Fig. 2. Responses for the UC and CC problems.

saturating element in the control loop to satisfy the control constraints. There are two main objections to such a feedback law. First, the state space region which can be stabilized by the saturation feedback law may be smaller than the region which can be stabilized by admissible controls. Reference 21 contains a simple example where this may occur. Secondly, even if the initial state is driven to the origin by the saturation feedback law, the quality 
Table 1. List of some useful solution properties.

\begin{tabular}{cccccccc}
\hline & \multicolumn{7}{c}{ Maximum magnitudes } \\
\cline { 3 - 7 } Problem & $M$ & $x_{\max }^{1}$ & $x_{\max }^{2}$ & $x_{\max }^{3}$ & $x_{\max }^{4}$ & $u_{\max }$ & $\begin{array}{c}\hat{V}^{*}(i, a ; \mathcal{M}) \\
\left(\text { or } V^{*}(i, a)\right)\end{array}$ \\
\hline UC-MH- $l_{1}$ & 30 & 5.0 & 4.55 & 1.36 & 4.50 & 7.67 & 76.68 \\
UC-1H- $l_{2}$ & - & 5.0 & 2.70 & 1.29 & 2.66 & 4.56 & 149.3 \\
CC-MH- $l_{1}$ & 30 & 5.0 & 1.62 & 1.00 & 1.56 & 1.00 & 83.27 \\
CC-MH- $l_{2}$ & 30 & 5.0 & 1.61 & 1.20 & 1.40 & 1.00 & 167.7 \\
\hline
\end{tabular}

of response may be poor. The second approach is to incorporate the control constraint directly in (1)-(3) and use the MH feedback law. It will be seen that this gives a much better quality of regulation than the saturation feedback law.

Discretizing the continuous-time system using a zero-order hold (period $T=1)$ gives

$$
\begin{aligned}
& x_{k+1}^{1}=x_{k}^{1}+x_{k}^{2}+0.5 u_{k}, \\
& x_{k+1}^{2}=x_{k}^{2}+u_{k}, \\
& y_{k}=x_{k}^{1}, \quad k \geq 0 .
\end{aligned}
$$

The saturation feedback law (SFL) is developed as follows. Solve an LQRP with $Q_{k}=1$ and $R_{k}=10^{-3}$ to get the linear feedback law $u_{k}=g x_{k}$, where $g=(-1.962,-1.962)$. Then, define

$$
u_{k}= \begin{cases}g x_{k}, & \text { if }\left|g x_{k}\right| \leq 1, \\ g x_{k} /\left|g x_{k}\right|, & \text { otherwise. }\end{cases}
$$

The MH feedback law (MHFL) is generated by the FOMP approach with $M_{k}=30, k \geq 0$, and the same quadratic cost function as in the LQRP.

A comparison of responses for a variety of initial conditions of sufficiently large amplitude shows that the MHFL leads to a well-damped response, whereas (49) leads to an oscillatory and very sluggish response. For instance, with $x_{0}=(15,-10)$, the number of zero-order hold periods required by the response to reach $\{x:\|x\| \leq 0.01\}$ is 26 and 72 , respectively, for the MHFL and the SFL. The failure of the SFL is not surprising, because the resulting control sequence differs widely from the optimal control sequence for the UC-IH- $l_{2}$ problem. Specifically, for $x_{0}=(15,-10)$, the UC-IH- $l_{2}$ problem gives $u_{\max }=9.81$.

The desirable behavior of the CC-MH- $l_{2}$ implementation in both examples suggests a promising approach to regulator system design. Wellknown techniques can be used to formulate an LQRP which has desirable 
characteristics such as good damping and robustness to modeling errors. Then, the large control amplitudes associated with the LQRP can be reduced by specifying a hard control constraint and implementing the CC-MH- $l_{2}$ system corresponding to the LQRP. For $M$ reasonably large and small state errors, the behavior of the CC-MH- $l_{2}$ and the UC-IH- $l_{2}$ systems should be nearly identical; thus, the CC-MH- $l_{2}$ implementation can be viewed as an effective nonlinear extension of the linear LQRP design.

\section{Appendix A: Proof of Theorem 3.2}

Let $i \geq 0$ and $\left\{\left(x_{k}, y_{k}, u_{k}\right)\right\}_{k \geq i}$ be a sequence triple that satisfies (1) and (5). Let $a=x_{i}$. For $k \geq i$,

$$
y_{k}=C_{k}\left(\Phi(k, i) a+\Phi(k, i+1) B_{i} u_{i}+\cdots+B_{k-1} u_{k-1}\right)+D_{k} u_{k} .
$$

Hence,

$$
\sum_{k=i}^{i+N_{0}-1}\left\|y_{k}\right\|^{2}=\sum_{k=i}^{i+N_{0}-1}\left\|C_{k} \Phi(k, i) a+L_{k, i} U_{i}\right\|^{2}
$$

where $U_{i}=\left(u_{i}, u_{i+1}, \ldots, u_{i+N_{0}-1}\right) \in R^{m N_{0}}$ and the matrices $L_{k, i}$ are appropriately defined using (50). Expansion of (51) gives

$$
\sum_{k=i}^{i+N_{0}-1}\left\|y_{k}\right\|^{2} \geq a^{\prime} G_{0}\left(i, N_{0}\right) a+2 a^{\prime} T_{i} U_{i},
$$

where

$$
T_{i}=\sum_{k=i}^{i+N_{0}-1} \Phi^{\prime}(k, i) C_{k}^{\prime} L_{k, i} \cdot
$$

By the uniform boundedness of $A_{k}, \exists \phi_{1}>0$ such that

$$
\|\Phi(i+j, i)\| \leq \phi_{1}^{j}, \quad i \geq 0, j \geq 0 .
$$

Because of (53) and the uniform boundedness of $B_{k}, C_{k}$, and $D_{k}$, there exists $\phi_{2}>0$ independent of $i$ such that $\left\|T_{i}\right\| \leq \phi_{2}$. Also, by (16) and (52),

$$
\sum_{k=i}^{i+N_{0}-1}\left\|y_{k}\right\|^{2} \geq \mu_{0}\|a\|^{2}-2 \phi_{2}\|a\|\left\|U_{i}\right\| .
$$

For $0 \leq \delta \leq 1$, we then have, by (54),

$$
\begin{aligned}
\left(\sum_{k=i}^{i+N_{0}-1}\left\|\left(y_{k}, u_{k}\right)\right\|\right)^{2} & \geq \sum_{k=i}^{i+N_{0}-1}\left\|\left(y_{k}, u_{k}\right)\right\|^{2} \geq \delta \sum_{k=i}^{i+N_{0}-1}\left\|y_{k}\right\|^{2}+\left\|U_{i}\right\|^{2} \\
& \geq \delta \mu_{0}\|a\|^{2}-2 \delta \phi_{2}\|a\|\left\|U_{i}\right\|+\left\|U_{i}\right\|^{2} \\
& \geq\left(\delta \mu_{0}-\delta^{2} \phi_{2}^{2}\right)\|a\|^{2} .
\end{aligned}
$$

Choose $\delta=\min \left(1, \mu_{0} / 2 \phi_{2}^{2}\right)$ and let $p_{0}=\sqrt{\delta \mu_{0} / 2}$ [note that $\delta \mu_{0} / 2 \leq$ $\left.\left(\delta \mu_{0}-\delta^{2} \phi_{2}^{2}\right)\right]$ to get $(17)$. 


\section{Appendix B: Existence Theorem}

Consider the optimal control problem, Problem $\mathscr{P}$, of minimizing

$$
J=\sum_{k=i}^{\infty} \mathscr{H}_{k}\left(x_{k}, u_{k}\right)
$$

subject to

$$
x_{k+1}=f_{k}\left(x_{k}, u_{k}\right), \quad\left(x_{k}, u_{k}\right) \in W_{k}, \quad k \geq i, \quad x_{i} \in T_{i} .
$$

A sequence pair $\left\{\left(x_{k}, u_{k}\right)\right\}_{k \geq i}$ is admissible to $\mathscr{P}$ if it satisfies (57). The following theorem gives conditions under which $\mathscr{P}$ has a solution.

Theorem 10.1. Assume that $T_{i}$ is compact and, for each $k \geq i$, the following conditions hold.

(a) $W_{k}$ is closed;

(b) $f_{k}: W_{k} \rightarrow R^{n}$ is continuous;

(c) $\mathscr{H}_{k}: W_{k} \rightarrow R$ is lower semicontinuous and nonnegative;

(d) there exists $\phi_{k}: R^{m} \rightarrow R$ which satisfies: $\phi_{k}(u) \rightarrow \infty$, whenever $\|u\| \rightarrow \infty, \mathscr{H}_{k}(x, u) \geq \phi_{k}(u),(x, u) \in W_{k}$.

Also assume that:

(e) there exists an admissible sequence pair with a finite cost.

Then, $\mathscr{P}$ has a solution.

The existence result also holds if (d) is replaced by: $\left(d_{1}\right)$ there exists a compact set $\Omega_{k} \subset R^{m}$ such that $\left\{u:(x, u) \in W_{k}\right\} \subset \Omega_{k}$.

Except for minor changes in notations, Theorem 10.1 is directly taken from Ref. 16: in particular, see Theorem 1 and conditions $\left(d_{1}\right)$ and $\left(d_{3}\right)$ of Theorem 2.

\section{References}

1. Bertsekas, D. P., Dynamic Programming and Stochastic Optimal Control, Academic Press, New York, New York, 1976.

2. KwakernaAk, H., and Sivan, R., Linear Optimal Control Systems, WileyInterscience, New York, New York, 1972.

3. Kleinman, D. L., An Easy Way to Stabilize a Linear Constant System, IEEE Transactions on Automatic Control, Vol. AC-15, p. 692, 1970.

4. ThOmas, Y., and BARRAUd, A., Commande Optimale à Horizon Fuyant, Revue RAIRO, Vol. J1, pp. 126-140, 1974.

5. ThOmAs, Y. A., Linear Quadratic Optimal Estimation and Control with Receding Horizon, Electronics Letters, Vol. 11, pp. 19-21, 1975. 
6. Kwon, W. H., and Pearson, A. E., A Modified Quadratic Cost Problem and Feedback Stabilization of a Linear System, IEEE Transactions on Automatic Control, Vol. AC-22, pp. 838-842, 1977.

7. KWON, W. H., and PEARSON, A. E., On Feedback Stabilization of Time-Varying Discrete Linear Systems, IEEE Transactions on Automatic Control, Vol. AC-23, pp. 479-481, 1978.

8. Kwon, W. H., Bruckstein, A. M., and Kailath, T., Stabilizing StateFeedback Design via the Moving Horizon Method, International Journal of Control, Vol. 37, pp. 631-643, 1983.

9. KeERTHi, S. S., and Gilbert, E. G., Moving-Horizon Approximations for a General Class of Optimal Nonlinear Infinite-Horizon Discrete-Time Systems, Conference on Information Sciences and Systems, Princeton, New Jersey, 1986.

10. KeERTHI, S. S., Optimal Feedback Control of Discrete-Time Systems with StateControl Constraints and General Cost Functions, PhD Dissertation, University of Michigan, Ann Arbor, Michigan, 1986.

11. KnUdSEN, J. K. H., Time-Optimal Computer Control of a Pilot Plant Evaporator, Proceedings of the 6th IFAC World Congress, Part 2, pp. 4531-4536, 1975.

12. DeVlieger, J. H., Verbruggen, H. B., and Bruijn, P. M., A Time-Optimal Control Algorithm for Digital Computer Control, Automatica, Vol. 18, pp. 239244, 1982.

13. Gutman, P. O., On-Line Use of a Linear Programming Controller, IFAC Software for Computer Control, Madrid, Spain, pp. 313-318, 1982.

14. Willems, J. L., Stability Theory of Dynamical Systems, Thomas Nelson and Sons, London, England, 1970.

15. Kalman, R. E., and Bertram, J. E., Control System Analysis and Design via the Second Method of Lyapunov, II: Discrete-Time Systems, Transactions of the ASME, Journal of Basic Engineering, Vol. 82, pp. 394-400, 1960.

16. Keerthi, S. S., and Gilbert, E. G., An Existence Theorem for Discrete-Time Infinite-Horizon Optimal Control Problems, IEEE Transactions on Automatic Control, Vol. AC-30, pp. 907-909, 1985.

17. KeERThi, S. S., and Gilbert, E. G., Optimal Infinite-Horizon Control and the Stabilization of Discrete-Time Systems: State-Control Constraints and Nonquadratic Cost Functions, IEEE Transactions on Automatic Control, Vol. AC-31, pp. 264-266, 1986.

18. HWAnG, W. G., and SCHMITENDORF, W. E., Controllability Results for Systems with a Nonconvex Target, IEEE Transactions on Automatic Control, Vol. AC-29, pp. 794-802, 1984.

19. Barrodale, I., and Roberts, F. D. K., Solution of the Constrained $l_{1}$ Linear Approximation Problem, ACM Transactions on Mathematical Software, Vol. 6, pp. 231-235, 1980.

20. LAwson, C. L., and HANSON, R. J., Solving Least Squares Problems. PrenticeHall, Englewood Cliffs, New Jersey, 1974.

21. Gutman, P. O., and Hagander, P., A New Design of Constrained Controllers for Linear Systems, IEEE Transactions on Automatic Control, Vol. AC-30, pp. 22-33, 1985. 